\title{
EL DISCURSO POLÍTICO DE OPOSICIÓN COMO ELEMENTO DE ADIESTRAMIENTO POLÍTICO
}

\author{
OPPOSITION POLITICAL DISCOURSE \\ AS AN ELEMENT OF POLITICAL TRAINING
}

Frank MEJía GUZMÁN*
Recibido: 20 de septiembre de 2017 - Aceptado: 20 de enero de 2018 - Publicado el 31 de diciembre de 2018 DOI: $10.24142 /$ raju.v13n27a8

\section{Resumen}

La comprensión del análisis del discurso político de oposición lo haremos en su momento, bajo el enfoque argumentativo, utilizando elementos de comprensión hermenéutica o de un estructuralismo que nos posibilite ahondar en esa herramienta de poder que tienen los gobiernos, la oposición, o el mismo ciudadano de a pie para lograr intervenir pragmáticamente el ejercicio político mediatizado. Para lograr comprender las dinámicas de un discurso que, por un lado busca establecer posición contraria a las directrices del gobierno de turno para luego, y por vía de ese mismo discurso, terminar delimitando la manera como deberíamos comprender lo político, la manera como

\footnotetext{
* Filósofo de la Universidad de Antioquia, Medellín (Colombia); Maestría en Estudios Políticos, Universidad Nacional de Colombia, Sede Medellín (Colombia). Correo electrónico: frmejiagu@ unal.edu.co
} 
deberíamos percibir el otro en oposición, ese otro que en el momento propio de la mediatización del discurso adquiere la piel del gobierno de turno, por cuanto es el gobierno en ejercicio. La intención es de alguna manera demostrar cómo el discurso político de oposición no es otra cosa que un interés por lograr permear la mirada de aquel que, sin elementos teóricos, intenta establecer su propio análisis político desde lo cotidiano; en otras palabras, el discurso como herramienta de adiestramiento político.

Palabras clave: análisis del discurso político de oposición, ciudadano, oposición, gobierno.

\section{Abstract}

The understanding of the analysis of the opposition's political discourse will be done at the time under the argumentative approach, using elements of hermeneutical understanding or a structuralism that allows us to delve into that tool of power that governments, the opposition, or the ordinary citizen have to achieve intervene pragmatically the mediated political exercise. To understand the dynamics of a discourse that on the one hand seeks to establish a position contrary to the guidelines of the current government and then, through that same discourse, to finish delimiting the way we should understand the political, the way we should perceive the other in opposition, that other one that at the proper moment of the mediatization of the discourse acquires the skin of the government of the day, because it is the government in office. The intention is in some way to demonstrate how the political discourse of opposition is nothing other than an interest in achieving permeate the gaze of those who, without theoretical elements, tries to establish his own political analysis from the everyday, in other words, discourse as a tool for political training.

Keywords: Analysis of Opposition Political Discourse, Citizen, Political, Opposition, Government. 


\section{PROEMIO}

Este ejercicio crítico de análisis sobre la oposición al gobierno de turno, tiene como contexto dos elementos cruciales que determinarán los límites bajo los cuales nos permitiremos precisamente hacer la disquisición. Por un lado, está el fenómeno práctico y discursivo de un tratado de paz en Colombia entre el gobierno y las Fuerzas Armadas Revolucionarias de Colombia (FARC), con una intención política primaria, como es la terminación del conflicto armado; en segundo lugar, la discursividad de oposición, pero en particular una que discurre dentro de lo que en su momento ha sido llamado, por el Centro Democrático, "un golpe a la democracia", y que se acuñó desde lo religioso, como paradigma importante para lograr permear, de manera más eficaz, las mentes de aquellos que antes que educación política acceden, desde la fe dogmática, a la percepción de "lo maligno", es decir, un antagonismo discursivo que tiene como base un deber ser democrático de paz, sesgado por un deber ser de lo bueno y correcto religioso, un tratado de paz que se expone como la acción de un Estado que terminaría congraciándose con aquellos que a la luz de Dios son los pecadores; esto es, una acción evidente de contradicción democrática.

Haremos un rastreo de ciertos momentos puntuales, no sistemáticos, ni conexos, de aquellas afirmaciones de los protagonistas de la oposición, que revelarán el proceder particular, beligerante y específico de un discurso político catolizado, a manera de prueba, de que, en su momento, la oposición abandonó lo crítico para adentrarse en lo dogmático, como elemento de rivalidad política.

Asunto evidente en el hecho fáctico de un uribismo opositor aledaño con la Misión Carismática Internacional que, por su volumen considerable de feligresía, pudo ejercer gran poder político de discrepancia, sin contar con actores políticos militando en dicha iglesia y al mismo tiempo con protagonistas en el Congreso de la República, desde la bancada del Centro Democrático: el senador Orlando Castañeda y la representante Esperanza Pinzón. Asunto este que comienza a dibujar el alcance de poder que tendría el discurso religioso de oposición al gobierno, que logró, en su momento, una victoria, si bien no holgada sí significativa de unos votantes que terminaron escuchando y pensando lo que el discurso uribista proclamaba a diestra y siniestra, apelando a concepciones religiosas que terminaron sesgando la mirada de aquel que votaría por la implementación o no de un tratado de paz. 
Los elementos, bajo los cuales nos permitiremos pensar el tema de la oposición, serán aquellos de la expresión del discurso oral, las expresiones antagónicas arrojadas en diversos contextos, pero siempre conflictivos, de una oposición mediatizada y, por ende, de gran impacto en una comunidad religiosa y poco conocedora de lo político, pero sí dogmática, que comprendía muy bien elementos de la fe que le eran más cercanos que los posibles argumentos jurídicos bondadosos de un potencial tratado que terminaría la violencia en Colombia, que acontece, en no pocas ocasiones, fundamento para la perorata firme de un partido, o de un individuo en ejercicio político, que termina erigiendo su respuesta o afirmación como pensamiento complejo de adversario, demostrando que en Colombia la distancia entre la religión y la política no existe, antes bien, se ha vuelto confusa.

Si bien, se supone que al lado del presidente Juan Manuel Santos estaría la Iglesia católica, intentando no hacer un ejercicio específico de participación política, pero acorde con lo que sustenta discursivamente un tratado de paz, en términos del amor al prójimo y el perdón, resulta bastante extraño la evidente distancia que tomaron los católicos al respecto; que antes que sumarse a las disposiciones en su momento del papa Francisco se unieron a las disposiciones de otras iglesias como la evangélica.

"Tengo que decir que el presidente (Juan Manuel) Santos está arriesgando todo por la paz, pero veo también otra parte que está arriesgando todo para continuar la guerra. Esto hiere el alma". ${ }^{1}$

Las palabras del pontífice apuntan a la necesidad de cercanía de la Iglesia católica al proceso de paz y, por ende, a la necesidad de apoyar el tratado en el plebiscito. Es extraño lo que se evidenció, por cuanto el acontecimiento fue totalmente contrario a lo que el papa señaló en no pocas ocasiones. Los creyentes católicos terminaron avalando un derrotero opositor, que acudía más a lo que se establecía en alocuciones de la Iglesia evangélica, acompañante del expresidente Álvaro Uribe Vélez y diametralmente ajena a la propuesta del gobierno.

Así, la victoria del No solo dejó en evidencia una oposición que supo recoger los niveles de frustración del pueblo y sus temores más infundados como individuos religiosos, antes que como sujetos inmersos en el conflicto armado. Quizá, y por el momento, sea posible señalar que ello se dio en la

1 Las palabras del pontífice se dieron durante el encuentro que tuvo el 26 de septiembre con una delegación del Congreso Judío Mundial, en su residencia en El Vaticano. 
dinámica alienadora de los medios, que exponían, de todas las maneras posibles, a un gobierno condescendiente, y que no purgaría las faltas de aquellos que tanto daño le habían hecho al pueblo. Es decir, nada del discurso católico que vela por el amor al prójimo, o la atenta y voluntaria exposición de la otra mejilla, hizo eco en la mayoría de los feligreses que irían a refrendar el tratado en el plebiscito.

\section{EL ACONTECIMIENTO}

Varios son los elementos que convergen en este momento de contingencia política en nuestro país. Después de 52 años de conflicto con el grupo guerrillero de las FARC, con cifras escalofriantes de guerra civil, como 225.000 muertos, 30.000 secuestrados y siete millones de desplazados, el actual presidente Juan Manuel Santos comienza contactos con el grupo insurgente en el 2010, para llegar a un primer acuerdo en septiembre de 2016. Acuerdo que sería negado en las urnas en un plebiscito el 2 de octubre.

El acontecimiento de la negación del acuerdo obedeció a una oposición acérrima que se alejó de lo político, y sustentó su diatriba detractora en lo religioso. Una oposición que antagónicamente esgrimió, en muchas ocasiones, oratorias y tesis críticas bastante alejadas del marco de lo político de un tratado de paz con las FARC; una oposición discursiva altamente compleja cuando, en procura de discutir el asunto de la paz, se terminó señalando temas como la educación de género. Asunto este que se presentó - si tenemos oídos críticos- bastante distante de lo que realmente se intentaba refrendar. La educación de género, como bandera discursiva de oposición, fue una de las más utilizadas, y cabe decir, una de las más eficaces si nos permitimos observar que fue un argumento que ganó gran atención del potencial votante en el plebiscito. Así, la intención de un gobierno por educar en la diferencia fue traducida por las élites religiosas protestantes o de ultraderecha católica, en voz del que fuera el procurador Alejandro Ordoñez, como una dinámica política contraria a la familia y al devenir de un Estado catolizado.

Así comienza entonces a dibujarse el modo como acontecería la crítica opositora al tratado de paz.

En una entrevista con "La W Radio", Ordóñez dijo que el "enfoque de género" de los acuerdos no está planteado en un solo sentido, en el documento que surgió tras las negociaciones entre el Gobierno Nacional y la guerrilla de las FARC en La Habana. "Por una parte", señaló, "está relacionado con la no discriminación y el reconocimiento y la promoción de los 
derechos de la mujer. Es indudable que ese alcance, ese sentido, no tiene ninguna objeción", dijo. "Pero hay otro sentido, que aparece con los contenidos que se le reconocen ideológicamente hoy a la noción género", añadió el exprocurador, y señaló: "La noción género, la novedad, es precisamente que este no tiene relación con el sexo, que no se nace hombre, ni mujer, sino que se hace. Eso implica toda una nueva antropología que va a impactar políticas públicas" ("Ordóñez dice que enfoque de género en acuerdos "tiene dos sentidos"”, 2016).

Como podemos ver, la oratoria, que discrepa desde asuntos que ocupan más al ser religioso que al ser político, no se asoma como nueva en nuestro devenir político, antes bien, es tan solo un resultado de nuestra propia historia. Es decir, la oposición religiosa-discursiva tiene una cuna histórica sin la cual el devenir de oposición política en la actualidad no obedecería a ese particular proceder. Así, este acontecimiento político de oposición está cimentado en una patria que se catolizó en 1800 por Miguel Antonio Caro, y se fortaleció con Rafael Núñez, quien hizo posible la regeneración como movimiento de una carrera política ultraconservadora emanada de un gran fervor católico, hasta el punto de promover y consolidar un partido político con dicha orientación de fe. Dicha condición religiosa lo hizo levantar su propuesta política en contra de los liberales radicales, para así lograr erigir una constitución con carácter definitivamente católico.

Solo de manera superficial, ya podemos permitirnos pensar por qué las incomodidades, desde entonces, redundan en dos protagonistas de lo político: el Estado religioso y el Estado laico, pensamiento este que, a su vez, y como lo señalaba Carl Schmidt, comenzará a dibujar la dinámica de amigos y enemigos. Así, este asunto de las molestias de los religiosos se da bajo el contexto de una política de reforma educacional, que comienza a crear una ampolla en la conciencia de algunos sectores ultrarreligiosos. La incomodidad entonces responde, por ejemplo, a políticas de Estado, como la exposición del aborto en posibilidades legales, una legitimidad potencial de la adopción de parejas del mismo sexo, así como el matrimonio entre parejas homosexuales, una política liderada por el Ministerio de Educación en la posibilidad abierta de incluir en los colegios unas prácticas que legitimaran la convivencia vigilada por ese estado de sujetos no heterosexuales. Todo este tema va en contra de las doctrinas ultrarreligiosas, que propenden por desarrollar su devenir devoto amparado y garantizado por un Estado que se debate entre lo laico y lo religioso. De ahí la contradicción, cuando aquellos que ejercen lo burócrata gubernamental terminan liderando proce- 
sos políticos, antes que, como funcionarios del gobierno, erigiéndose como líderes carismáticos religiosos. Solo como momento ejemplificador está ese discurso de respuesta que presenta el señor Alejandro Ordoñez cuando, por disposición de la Suprema Corte, es destituido de su ejercicio como Procurador General de la Nación. Frente a la destitución el procurador responde: "Simplemente, la acepto con resignación cristiana".

Pues bien, como podemos ver la tensión es evidente, una oposición que fluctúa entre oponerse a un tratado de paz, que ven como contradictorio a su ejercicio religioso acrítico y de total obediencia a lo jerárquico de la iglesia, y una oposición mínimamente crítica en lo político. Hecho que no deja al interlocutor político más que una nebulosa sensación de que se están mezclando piedras con bananos. Sin embargo, este que escribe no deja de sorprenderse por esa facilidad mimetizadora del discurso fascista católico, que en aras de un orden ideal de paz religiosa termina avalando la necesidad de la guerra.

La guerra nunca es algo bueno, pero algunas veces es algo necesario. En un mundo lleno de gente pecadora, la guerra es inevitable. Algunas veces la única manera de evitar que la gente pecadora haga un gran daño es yendo a la guerra contra ellos (Romanos 3: 10-18, 2016).

\section{EL MENSAJE}

"Yo creo que por lo menos dos de los seis millones de votos (que tuvo el 'No') fueron de congregaciones cristianas, evangélicas y católicas que salieron a votar en defensa de la familia, como si la familia estuviera en riesgo". ${ }^{2}$

Es curioso cómo, en voz de protagonistas beligerantes como Álvaro Uribe Vélez, Alejandro Ordoñez y, en su momento, el pastor de la iglesia evangelista Eduardo Cañas, un discurso de perdón y reconciliación, de amor al prójimo, toma tintes excluyentes; es decir, el prójimo dejó de ser el otro para convertirse en algunos, como el perdón acontece en determinados escenarios, como la curia, que propendería por el respeto y la reconciliación, acude a lo más soterrado de un discurso fundamentalista de la religión para acontecer, en distancias, con aquel de izquierda, con el guerrillero como sujeto en observancia, la venganza y el castigo. Convergen discursos ajenos de

2 Palabras del senador oficialista Roy Barreras, dos días después del resultado del plebiscito. 
lo estrictamente religioso en función de un sujeto político. La posibilidad de adopción, el respeto por la diferencia de género y la educación en la diferencia terminan mezclándose con el odio por los victimarios, que son expuestos en una necesidad de purga. La intervención efectiva, activa y reaccionaria de un sector que de alguna manera debería mantenerse al margen del devenir de lo político, en tanto este no involucrara especificidades de contradicción en lo religioso, ahora se convierte en un grupo de ciudadanos que se reagrupan con un enemigo común, la guerrilla, el homosexual, el aborto, etc.

En principio, el discurso de oposición se podría pretender como arengas al interior de una iglesia, o molestias entre sujetos comunes de una misma fe, pero pasó a ser una locución íntima de opinión, expresiones de individuos en ejercicio de sus cargos políticos, para luego, y dentro de la dinámica de las comunicaciones, terminar permeando al ciudadano que acude a ser informado por redes sociales, a las cuales no se les hace ningún tipo de filtro, y que por el contrario terminan depurando el proceder crítico del feligrés desprovisto de análisis o regulación inteligente. Así, el adepto religioso, que se sabe acrítico, por cuanto su proceder religioso así lo conmina, escucha la arenga del Twitter y no la traduce, solo acude a ella para hacerla multiplicar entre iguales. "Si el pastor lo dice debe ser verdad" y si

lo dice de esa manera debe tener aún mayor peso. "El destino de Colombia no puede quedar en manos de Satanás". ${ }^{3}$

Hagamos, por lo pronto, un ejercicio escrutador de un pastor de la Iglesia Manantial (protestante), que se opone al tratado de paz desde una observancia estrictamente religiosa; en una página que sale en su momento, llamada Pastores de Bogotá unidos por la paz, pero en contra del plebiscito, se argumenta la oposición desde la doctrina religiosa antes que desde la crítica política de oposición.

Con respecto al plebiscito para la refrendación del tratado, la Iglesia protestante se refiere a este:

Es allí (el voto del plebiscito) donde el pueblo de Colombia y, en especial, la Iglesia de Jesucristo en esa nación entran en escena puesto que la aprobación de este Acuerdo de "paz" incluye otros temas de gran importancia como lo es la identidad de género.

3 Afirmó en prédica el pastor de la Iglesia Manantial, Eduardo Cañas Estrada, al pedir votar NO al referendo". Afirmación que repitió por medio de un mensaje de Whatsapp que se viralizó. 
La identidad, ideología o perspectiva de género, como también se le dice, "tiene como base la promiscuidad sexual, es decir, la legalización de prácticas de las relaciones sexuales con varias parejas o grupos. Al legalizarse las violaciones van a surgir y no pasará de un llamado de atención porque ya se volvió política de Estado. La promiscuidad es una relación poco estable con varias personas", dijo el apóstol Eduardo Cañas Estrada, fundador de la Iglesia Manantial de Vida Eterna en Bogotá, Colombia ("Pastores de Bogotá unidos por la paz pero en contra de plebiscito", 2016).

Es evidente que la oposición al tratado de paz abandonó, no en pocos momentos, la circunstancia primigenia de oposición para ocuparse de temas que, si bien podría estar orbitando el tratado, solo lo harían de manera satelital y nunca de manera nuclear. La oposición entonces buscó remover una moral religiosa para lograr adeptos opositores de un tratado de paz, que versa sobre la intención de finalización de un conflicto armado antes que la legitimación del sujeto y sus prácticas sexuales.

"Como cristianos queremos que se acabe la guerrilla, pero que no se acabe la familia... con la aprobación total del proyecto se acabaría la familia”, denunció Cañas. Informó, además, que el primer propósito de la identidad de género es destruir a la familia como Dios la creó. El segundo propósito que tiene es ir en contra de la naturaleza creada por Dios, "esto no es homofobia ni condenarlos a ellos [la comunidad LGTBI], sino condenar el pecado que ellos hacen. Si el plebiscito no gana, el proyecto no va para Colombia" ("Pastores de Bogotá unidos por la paz pero en contra de plebiscito", 2016).

Podríamos hacer una y otra vez el ejercicio de exégesis sobre el mensaje de las pastorales religiosas, y nos daremos cuenta de que todas ellas responden a dibujar un tratado de paz que propende, según ellos, no por la desmovilización y la terminación de un conflicto de más de medio siglo, sino, antes bien, por la legitimación, por parte del Estado, de una sociedad promiscua y apta para lo que ellos llaman el pecado.

"El Señor está levantando una Iglesia activa en Colombia para que se alce en oración y unión contra todo lo que sea contrario a Él’. ${ }^{4}$

4 Frase aludida al pastor Eduardo Cañas Estrada, al señalar el proceso de paz y al gobierno Santos. 
Él, termina siendo entonces, para el opositor religioso, el enemigo del pueblo creyente. Aparece, en este espectro de oposición religiosa, el asunto del pecado, acudiendo a un escenario de lo político. Un pecado que para el laico puede no concurrir a nada relevante, pero no así para el practicante fervoroso. El pecado será la manera como el individuo practicante de la fe religiosa, sea católica o evangélica, traduce lo fatal; y acá lo fatal responde a un Estado que, por vía de un tratado, intenta legitimar lo contradictorio a su fe. Así mismo, para el practicante religioso el asunto de la guerra puede resultar altamente conveniente, dado que es en ese contexto donde se ha eliminado la plaga de pecadores. Una de las fortalezas discursivas que tiene la religión es la de dejar de manera tácita, en algunos momentos y de manera explícita en otros, la condición de amenaza constante en un mundo que, por sí mismo, intenta coaccionar al sujeto para que se distraiga del camino de la fe. La distracción son las FARC, es el ESTADO condescendiente, y por ende figura de pecado. Los discursos de oposición que percibimos una y otra vez no dejan de dibujar los enemigos a quienes hay que destruir, y terminan indirectamente construyendo los buenos (amigos) y señalando los malos (enemigos).

\section{DETRÁS DE LA INTENCIÓN}

Detrás del discurso de oposición existe una intención oculta para aquel lector cotidiano de lo político. Saben de antemano que el feligrés tiene, como proceder de escucha, la no indagación, el sometimiento a la figura de poder. Si bien, hay una aparente intención por lograr un tratado depurado que propenda por el amor al prójimo, la restitución y respeto por la víctima, el amparo de los desprotegidos del conflicto, es decir, un tratado que funcione para "dar a cada quien lo suyo", esconde, de manera muy probablemente volitiva, la idea de desestabilizar un proceso en favor de una óptica populista de intereses disfrazados como pueriles en pro de alcanzar protagonismo político, adiestrar al votante que elegirá el próximo presidente del país. Ordoñez se vislumbra candidato presidencial, Álvaro Uribe Vélez continúa carrera de desprestigio para fortalecer los intereses de su candidato presidenciable a futuro, un partido de oposición recalcitrante que quiere desviar la mirada de su propio y errático proceder político, evidenciado en sus militantes de partido.

El discurso opositor religioso se deja oír constantemente, aludiendo articulados inexistentes, señalando potencialmente un incumplimiento, 
apuntando, en su momento, intereses negociados para la salida de aquel contradictor y así lograr poner en la superficie a un mártir político que luego recogerá frutos por su sacrificio.

"Se acaba de cumplir el primer pacto de La Habana: la expulsión del procurador". 5

Así, el discurso de oposición tuvo como adeptos oídos no escrutadores, sumisos a una exposición mediática que levanta polvo y que evade en principio el debate, para luego acudir a señalar que siempre fue su intención. Un procurador resignado como lo manda la iglesia, presentándose como perseguido político, desviando la mirada de las causas legítimas de su destitución, apareciendo de manera elocuente, dibujando para los medios internacionales (CNN) las condiciones supuestamente ilegales de su destitución, exponiéndose como el mesías de la verdad; el elegido.

\section{ALIANZA PARA LA OPOSICIÓN}

Dentro de esa oposición de tinte doctrinal religioso se ha logrado un fortalecimiento importante de sus filas, a partir de alianzas tremendamente beligerantes en el espectro de la política colombiana. En su momento se unieron: el procurador Alejandro Ordóñez Maldonado, los voceros de la Iglesia católica y las organizaciones cristianas del país, para oficializar una alianza en contra del matrimonio gai en Colombia; si bien esta alianza se dio en el marco de la discusión por la legitimación del matrimonio entre personas del mismo sexo, dicha alianza continúa su camino opositor con respecto al tratado de paz, al que tildan de acudir a legitimar, por medio del marco legal del mismo, dinámicas no religiosas que para la iglesia y los sectores aliados van en contra de la familia. Así mismo, los senadores Edgar Espíndola Niño (PIN) y Claudia Wilches Sarmiento (La U) promovieron la creación del bloque antimatrimonio gai. Vale la pena recordar que Espíndola y Wilches representan, en el Congreso, a importantes organizaciones cristianas, abiertamente opositoras al matrimonio homosexual y que terminaron esgrimiendo, bajo la misma diatriba moral, un discurso de oposición al tratado.

5 Declaración del Procurador General de la Nación, Alejandro Ordóñez Maldonado (7 de septiembre de 2016) en respuesta a la decisión del Consejo de Estado de su destitución. 
Es inconstitucional el reconocimiento de familia a personas del mismo sexo, $[\ldots]$ es de recordar que el Congreso de la República fue elegido por los colombianos para que los represente haciendo las leyes, ejerciendo control político y modificar la Constitución Nacional de ser necesario, pero vemos con preocupación cómo la Corte está legislando por la vía de la interpretación (Espíndola, 2016).

Es importante señalar que la oposición acérrima de estos sectores ultraconservadores no se hace necesaria, y simplemente, desde su posición fervorosa, logran, desde el marco jurídico, legitimar posiciones personales. Por ejemplo, la base legal que utilizara Ordóñez en su momento para ejercer, fácticamente, su oposición es la alusión al artículo 42 de la Constitución, donde se hacen varias salvedades para la formalización de la familia en Colombia. "Artículo 42. La familia es el núcleo fundamental de la sociedad. Se constituye por vínculos naturales o jurídicos, por la decisión libre de un hombre y una mujer de contraer matrimonio o por la voluntad responsable de conformarla" (Corte Constitucional, 2011, título 1).

En este contexto, y de manera directa, el Centro Democrático pidió se sumara a la campaña adelantada por el No al plebiscito, y que como pudimos evidenciar históricamente tuvo sus frutos. "Nos parece que la llegada del exprocurador Ordóñez sería muy importante porque ha sido un defensor del Estado de derecho y por eso necesitamos que esté con nosotros" (s. d.). Las alianzas, entonces, se construyeron de manera efectiva, si nos atenemos al resultado del plebiscito, alianzas eficaces entre el Centro Democrático y Ordoñez en la procuraduría, y luego como exprocurador; también la que se dio entre el Centro Democrático, las comunidades jóvenes religiosas y el clero como Iglesia católica. La alianza entre el partido Centro Democrático y la Misión Carismática Internacional. Alianzas que se soportan en el marco de lo legal y con intenciones formales de oposición, que terminaron siendo protagonistas de gran peso político.

\section{CAUSA DE LA EFICACIA OPOSITORA DEL DISCURSO}

La pregunta que surge, luego de haber habitado el triunfo de la oposición en el plebiscito, es, ¿por qué, precisamente este tipo de discurso de lo religioso, logró agrupar tal masa de adeptos?

Por un lado, habitamos la incredulidad histórica frente al gobierno de turno; ello le dio al discurso de oposición condiciones de exposición ha- 
bilitadas como viables y en necesidad, es decir, lo que se escuchaba era un ejercicio que hizo la crítica desde lo sagrado, y se abandonó a lo profano. La fuerza de convocatoria del discurso religioso se traduce una vez mediatizado, en circunstancias de flujo en términos de tiempo y de espacio como ningún otro; esto es, un pastor de iglesia que convoca a traducir sus palabras al otro, en un nicho que exige obediencia. Así mismo, se dio por parte de personajes altamente favorecidos por la opinión, dado que representan el clamor del pueblo, pero en particular de un pueblo que ve en esas figuras la ratificación de su fe, ello re-crea la sensación de gobernabilidad y poder desde la creencia de lo primitivo religioso. "Aquel que nos gobierna, es creyente".

El destino de Colombia no puede estar en las manos de Satanás, hemos peleado mucho por la familia de Colombia y sería muy triste que este plebiscito abriera las puertas de la destrucción familiar y a la degeneración de todo lo que Dios estableció, que fue el matrimonio entre un hombre y una mujer. ${ }^{6}$

Qué otro discurso podría haber sido más efectivo que sembrar en una población de creyentes en Colombia, donde ocho de cada diez se declaran religiosos, un tratado de paz que amenazaba su condición de fe, un tratado que se opone a lo dictaminado por Dios mismo en la Biblia.

Si bien, estas razones pueden carecer de fundamento y terminan erigiéndose como causas que no habitan la discusión y, por ende, que estaban en su momento permeadas por la fuerza del discurso de la creencia religiosa.

En profundidad, podríamos pensar que detrás de la causa de la fe está el que el discurso religioso de oposición tuviera figuras tan relevantes del quehacer político en el país, como el senador y expresidente Álvaro Uribe Vélez, que para muchos expone la figura de aquel que ha hecho el mejor ejercicio de gobierno en nuestro tiempo, y que lo hizo desde una figura representativa de aquel que gobierna como "Dios quiere".

Emulando a Carl Schmidt, Uribe representa al amigo, y todo aquel que erigiera como contrario sería asumido como enemigo. Individuos carismáticos que construyeron su crítica desde lo más íntimo que tiene el ser humano, su religiosidad. De ahí que los oídos santificados de los feligreses solo escucharan afablemente palabras que establecían que la oposición no

6 Frase dicha por Eduardo Cañas Estrada, en alocución para exhortar a sus feligreses al voto por el No frente al plebiscito. 
era en contra de la paz, sino en contra del proceso; ello terminaba legitimando, en aquel sujeto sumiso, una condición válida que lo convertía, para sí mismo, en un individuo actuando en diatriba pastoral y nunca como un enemigo de lo que definitivamente cualquiera querría: una paz estable y duradera.

\section{REFERENCIAS}

Corte Constitucional (2011). Sentencia C-577. Recuperado de http:// www.corteconstitucional.gov.co/relatoria/2011/C-577-11.htm

Espíndola, P. (2016). "Es inconstitucional el reconocimiento de familia a personas del mismo sexo": senador Espíndola. Recuperado de http:// www.senado.gov.co/historia/item/16602-es-inconstitucional-el-reconocimiento-de-familia-a-personas-del-mismo-sexo-senador-espindola

Horkheimer, M. (2003). Teoría crítica. Madrid: Amorrortu.

La Iglesia católica (2016). La Santa Sede. Recuperado de http://w2. vatican.va/content/vatican/es.html

Ordóñez dice que enfoque de género en acuerdos "tiene dos sentidos" (2016). El Tiempo. Recuperado de http://www.eltiempo.com/politica/ proceso-de-paz/ordonez-habla-de-ideologia-de-genero-en-acuerdos-depaz/16724937

Pastores de Bogotá unidos por la paz pero en contra de plebiscito (2016). Verdad y Vida. Recuperado de http://verdadyvida.org/ES/pastoresde-bogota-unidos-por-la-paz-pero-en-contra-de-plebiscito/

Romanos (2016). La Biblia. Recuperado de http://biblia.com/bible/ nvi/Rom\%203.10-18?culture=es 